\title{
In-silico screening of Schistosoma mansoni Sirtuin 1 inhibitors for prioritization of drug candidates
}

\author{
Raghvendra Singh ${ }^{1}$, Birendra Singh Yadav², Swati Singh ${ }^{1}$, Paras Nath Pandey ${ }^{3}$ and Ashutosh Mani ${ }^{2 *}$
}

\begin{abstract}
Schistosomiasis is a common, neglected parasitic disease caused by Schistosoma mansoni. Availability of two specific drug oxamniquine and praziquintel for treatment of the disease is a major concern. Recently NAD+ dependent lysine deacetylases have been identified as new drug targets in pathogens. Sirtuins are NAD+ dependent lysine deacetylases that are involved in a wide variety of vital cellular processes. Amongst them, members of sirtuin's class 1 proteins are considered to be main target of the drugs. Sirtinol and Salermide are two known inhibitors of Schistosoma mansoni Class1 sirtuin which is a protein with unknown 3-D structure. Here, we investigate molecular insights of interaction between modeled sirtuin 1 structure and it's inhibitors, that were derivatives of Sirtinol and Salermide, to prioritize them for their binding affinities with target. A detailed examination of absorption, distribution, metabolism and toxicity of these inhibitors has also been included in the study. Finally we found two derivatives of Sirtinol to be most appropriate drug candidates for Schistosomiasis.
\end{abstract}

Keywords: Intestinal schistosomiasis, Sirtuin 1, Sirtinol 1, Molecular docking, ADMET profiling

\section{Background}

Schistosomiasis, one of the most common parasitic diseases in developing countries, is caused by species of dioecious blood flukes belonging to the genus Schistosoma. After malaria, schistosomiasis is the most important tropical disease in terms of human morbidity. Under Schistosomiasis control initiative more than 40 million doses of Praziquantel have been dispensed in sub-Saharan Africa (Fenwick et al. 2009). Praziquantel is reported as the only drug used for mass treatment of schistosomiasis (Dömling and Khoury 2010) while Oxamniquine is a specific drug for Schistosomiasis mansoni (Cioli 1993; Fallon 1994). So, availability of the limited drug for the disease draws attention towards the search for new therapeutic targets as well as development of novel compounds to overcome the prospective threats from resistant strains of schistosomes (Doenhoff et al. 2008) that

\footnotetext{
*Correspondence: amani@mnnit.ac.in

${ }^{2}$ Department of Biotechnology, Motilal Nehru National Institute

of Technology Allahabad, Allahabad 211004, India

Full list of author information is available at the end of the article
}

have been already reported and characterized in endemic areas (Melman et al. 2009).

Recently NAD+ dependent lysine deacetylases (Histone modifying enzymes) have been identified as new drug targets in several pathogen (J Pierce et al. 2012). Sirtuin1 protein in Schistosoma mansoni, a member of NAD+ dependent deacetylases family which is phylogenetically unrelated to the $\mathrm{Zn}^{2+}$-dependent deacetylase (Frye 2000), has been targeted in assays designed to study the therapeutic effect of inhibitors (Lancelot et al. 2013). Sirtuin proteins have been classified into five different classes (I, II, III, IV and U), on the basis of presence of conserved motifs in their core domain (Religa and Waters 2012). Parasitic class I sirtuins, characterized by the GAGXSXXXGIPDFRS, PS/TXXH, TQNID and HG motifs (Religa and Waters 2012) have been extensively and successfully explored as antiparasitic targets (Vergnes et al. 2002). It has been reported that these proteins have vital role in parasite survival by catalyzing the deacetylation reaction of acetylated lysine residues of nuclear histones and other substrates, with NAD+ as a cofactor (Vergnes et al. 2002). Salermide, which induces

\section{至 Springer}

(c) 2016 Singh et al. This article is distributed under the terms of the Creative Commons Attribution 4.0 International License (http://creativecommons.org/licenses/by/4.0/), which permits unrestricted use, distribution, and reproduction in any medium, provided you give appropriate credit to the original author(s) and the source, provide a link to the Creative Commons license, and indicate if changes were made. 
cell death in S. mansoni by targetting both Sirt1 and Sirt2 (Lara et al. 2009), is a potential anticancer agent due to it's sirtuin inhibition property. The inhibition of sirtuins has been less explored for their therapeutic use against parasites. The molecular features of SmSirt2 as well as it use for the development of new targets for schistosomiasis were explored in a recent studies (Singh et al. 2015; Singh and Pandey 2015). In the present paper Sirt1 protein of S. mansoni has been used for the study. Due to unavailability of determined three dimensional structure of $S$. mansoni Sirt1 protein molecular insights of the inhibitor protein interaction or their participating residues are not known. Here we have modeled a 3-D structure of the protein by multi-template homology modeling. After that ten derivatives of salermide and sirtinol were screened against the modeled structure by docking. For sorting the inhibitors according to their druggability they were assessed on ADMET parameters.

\section{Methods}

Sequence retrieval and phylogenetic analysis

Sirt1 protein sequence of $S$. mansoni was obtained from Uniprot (Acession no. A6XDL2). Physicochemical properties were predicted by using ProtParam server (http://web.expasy.org/protparam/). BLASTp (Altschul et al. 1990) program was used to search similar protein sequences against non-redundant protein database in NCBI. The Sirt1 amino acid sequence was used as query sequence and identical amino acid sequences present in different species were selected for further study (Table 1). The Multiple Sequence Alignment of protein sequences was performed using ClustalW 2.0.10 program (Larkin et al. 2007). MEGA5.2 (Tamura et al. 2011) was used for constructing and analysing the phylogenetic tree. The neighbor-joining method (Saitou and Nei 1987) was used to get the information of evolutionary history. All the characters were having equal probability for transition. The 10,000 replicates of bootstrap consensus were taken to represent the evolutionary history of the taxa (Felsenstein 1985). Branches having less than 50 \% bootstrap replicates were sorted out. The percentage of replicate trees in which the associated taxa clustered together in the bootstrap test (1000 replicates) is shown next to the branches. The tree is drawn to scale with branch lengths in the same units as those of the evolutionary distances used to infer the phylogenetic tree. The evolutionary distances were computed using the Poisson correction method and are in the units of the number of amino acid substitutions per site. All positions containing gaps and missing data were eliminated from the dataset (complete deletion option). BioEdit 7.0.2 (Hall 1999) has been used to calculate the entropy.

\section{Multi-template homology modeling of Sirt1 protein}

PSI-BLAST (Altschul et al. 1997) algorithm was used against the Protein Data Bank (www.pdb.org) to search homologous sequences having 3D structure solved by experimental techniques. After PSI-BLAST search 50 protein structures were found then after three structures having PDB ID 2hjh, 4i5i and 4iao matching with different positions of query sequence were used as a template. Multi-template modeling was performed by MODELLER 9.10 (Šali and Blundell 1993). Homology modeling of was done by the steps: template selection from psi-blast, sequence multiple template alignment, multiple model building, evaluation of model, model refinement and model validation (Martí-Renom et al. 2000).

\section{Protein structure optimization, quality assessment and visualization}

MODELLER generated several preliminary models which were ranked based on their DOPE and GA scores. Models with low DOPE score were selected and stereo chemical property of each model was checked by PROCHECK. The model which having low DOPE score and least number of residues falling in disallowed region in Ramachandran plot was selected for further study. ProSA-Web server (Wiederstein and Sippl 2007) was used to check the quality of models, energy and stereochemical geometry.

Table 1 Comparison of DOPE score, quality factor determination through ERRAT and stereochemical property generated by Ramachandran plot of five models predicted through MODELLER

\begin{tabular}{lllllcc}
\hline & $\begin{array}{l}\text { Residues in most } \\
\text { favored regions }\end{array}$ & $\begin{array}{l}\text { Residues in additional } \\
\text { allowed region }\end{array}$ & $\begin{array}{l}\text { Residues in generously } \\
\text { allowed region }\end{array}$ & $\begin{array}{l}\text { Residue in disal- } \\
\text { lowed region }\end{array}$ & $\begin{array}{l}\text { DOPE Score } \\
\text { Overall quality } \\
\text { score errat }\end{array}$ \\
\hline Model1 & 0.858 & 0.125 & 0.009 & 0.009 & $-43,519.9$ & 53.971 \\
Model2 & 0.906 & 0.083 & 0.009 & 0.002 & $-44,363.7$ & 60.000 \\
Model3 & 0.875 & 0.112 & 0.009 & 0.004 & $-43,488.5$ & 48.381 \\
Model4 & 0.877 & 0.116 & 0.004 & 0.002 & $-43,591.1$ & 56.883 \\
Model5 & 0.891 & 0.096 & 0.011 & 0.002 & $-43,627$ & 55.052 \\
\hline
\end{tabular}




\section{Active sites prediction and ligand designing}

Active site in the protein model were identified by using DoGSite sever (Volkamer et al. 2012). Scoring was based on a linear combination of the three descriptors volume, enclosure and hydrophobicity for all the pockets present in the protein.

Sirtinol and Salermide are two known lead compounds against the Sirtuin Protein. Four derivatives of salermide were generated by using Zinc database on the basis of similarity. The four derivatives of sirtinol were constructed by Chemsketch11.0.[ACD/Structure Elucidator, version 11.0, Advanced Chemistry Development, Inc., Toronto, ON, Canada, www.acdlabs.com, 2008].

\section{Molecular docking}

Pre-docking steps were performed with the help of AutoDockTools 4.2 in which grid was generated near the active site residue Ala103 (GlyAlaGly domain) (Morris et al. 2009). Molecular docking was performed by Autodock 4.2. Flexible docking algorithm was used to check the interaction between ligand and protein. Average Grid points used in this docking were $(60,60,60)$ and centre of ligand was approximately as coordinates:

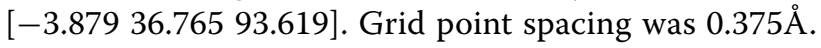
Lamarckian genetic algorithm (LGA) was used for docking.

\section{ADMET screening}

In order to rank the putative drug candidates on the basis of Absorption, Distribution, Metabolism, Excretion and Toxicity (ADMET) properties the structures of salermide, sirtniol and eight derivatives were done by using admetSAR (Cheng et al. 2012). First the structures of ligand PDB files were converted to SMILEs files by using Online SMILES Translator and Structure File Generator of National cancer Institute (http://www.cactus.nci. nih.gov) and these files were used as input for ADMET prediction.

\section{Results and discussion}

\section{Physicochemical properties}

The S. mansoni. Sirt1 protein is 568 amino acids long and was predicted to have a molecular weight of $63,262.3$ Daltons with an isoelectric point (pI) of 4.71. Sirt 1 has a Negative GRAVY index of -0.513 which is indicative of a hydrophilic and soluble protein.

\section{Homology modeling and structure validation}

The selected template protein's PDB IDs are $2 \mathrm{hjh}$, $4 \mathrm{i} 5 \mathrm{i}$ and 4iao. These template proteins showing 40, 46 and $46 \%$ identity with target protein sequence, respectively. The multiple template modeling was done by using these three protein structures.

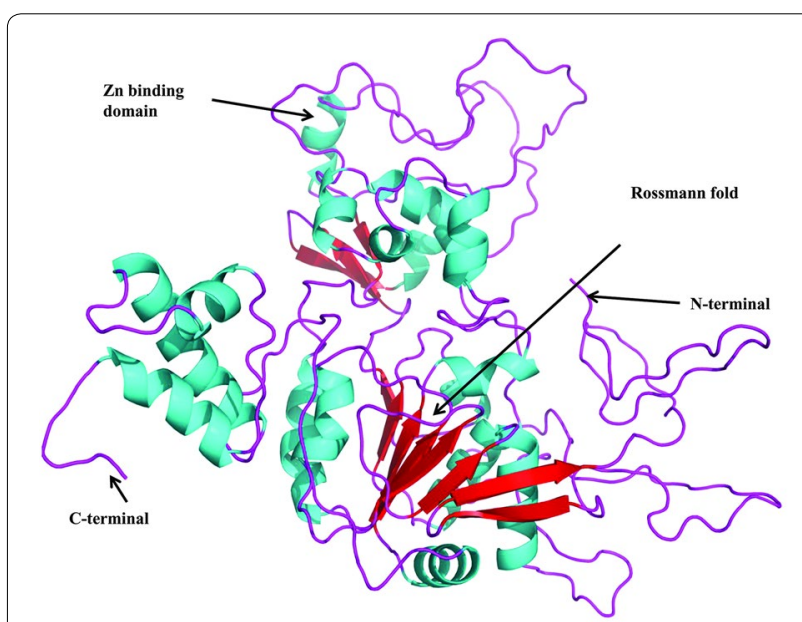

Fig. 1 3-D model of sirtuin 1 protein of Schistosoma mansoni predicted by multi-template modeling

For selection and validating the modeled structure (Fig. 1) obtained from the Modeller 9.13, DOPE score and Ramachandran plot was drawn using PROCHECK (Ramachandran plot has been shown in Additional file 1: Figure S1), quality score generated by ERRAT server were used. On the basis of DOPE score value (Table 1) model2 was selected for the further downstream analysis. Selected model subjected for validation step by`analyzing steriochemical property and quality score generated by ERRAT server. Steriochemical property and ERNET score of all the modeled structures are compared (Table 1). Ramachandran plot of Selected model are shown in Additional file 1: Figure S1 have the phi/psi angles of $90.6 \%$ residues are in the most favored region, $8.3 \%$ residues are in additional allowed region, $0.9 \%$ residues are in generously allowed region and $0.2 \%$ residues are in disallowed region (Additional file 1: Figure S1). Analysis suggested that model2 is best representative of Sirt1 with respect to Ramachandran plot and ERNET score and further selected for downstream analysis.

The overall protein quality and its structural deviation from the total energy were measured by Z-Score (Additional file 1: Figure S2). The black point in Additional file 1: Figure S2 represents the Z-score of the protein. Groups of structures determined from different source (NMR, X-ray) are distinguished by different color (NMR with dark blue and X-ray by light blue color). The plot of Z-Score represents value of the modeled protein of Schistosoma mansoni is (4.5) is located within the space of proteins related to NMR. The modeled protein's Z-Score is within the acceptable range ( -10 to 10 , negative Z-score are good and depend on length of protein).

Modeled protein has large number of insertion in both the terminals (Fig. 1). Explicitly blast tool was used to find 
sequence similarity of inserted regions but it did not give any kind of similarity with existing annotated sequences.

\section{Active site prediction}

The probable binding sites and other sub binding site were detected in the predicted model of sirt1 and analyzed in terms of their geometrical, as well as physico-chemical properties. The DoGSite Scorer predicts eighteen binding pockets in terms of calculated descriptors as shown in Table 2. The ligand generally forms favorable interactions with the binding sites having largest interacting cavity, so active pocket P0 can be predicted as potential binding pocket. Aminoacid composition descriptors calculate the values in term of non-polar amino acid ratio, polar amino acid ratio, positively charged amino acid ratio and negatively charged amino acid ratio and they are $0.45,0.34,0.22$ and 0.09 respectively. The most favorable binding site contains amino acids with high conserved residue score and amino acid residues: Ala, Arg, Asn, Asp, Cys, Gln, Glu, Gly, His, Ile, Leu, Lys, Met, Phe, Pro, Ser, Thr, Trp, Tyr and Val are responsible for constituting potential active-site of situin1 structure.

Table 2 Pockets and descriptors calculation for Sirtuin 1 model

\begin{tabular}{|c|c|c|c|c|c|}
\hline Name & Volume [Å & ]$^{3}$ Surface $[\AA ̊]^{2}$ & $\begin{array}{l}{ }^{2} \text { Lipo sur- } \\
\text { face }[\AA \AA]^{2}\end{array}$ & Depth [Å] & $\begin{array}{l}\text { Simple } \\
\text { score }\end{array}$ \\
\hline PO & 2117.23 & 2817.48 & 1667.61 & 27.66 & 0.6 \\
\hline P1 & 665.35 & 852.27 & 558.99 & 18.26 & 0.44 \\
\hline P2 & 605.27 & 765.08 & 445.91 & 16.5 & 0.33 \\
\hline P3 & 482.42 & 960.84 & 633.42 & 12.77 & 0.32 \\
\hline P4 & 442.11 & 793.97 & 462.03 & 16.59 & 0.24 \\
\hline P5 & 281 & 618.08 & 400.35 & 13.89 & 0.15 \\
\hline P6 & 241.21 & 180.08 & 175.7 & 18.94 & 0 \\
\hline P7 & 213.87 & 330.72 & 200.03 & 8.92 & 0.04 \\
\hline P8 & 196.15 & 322.3 & 206.77 & 10.56 & 0 \\
\hline P9 & 186.27 & 436.36 & 274.88 & 10.81 & 0 \\
\hline P10 & 183.57 & 233.86 & 187.31 & 10.71 & 0 \\
\hline P11 & 167.65 & 409.83 & 251.77 & 9.69 & 0.02 \\
\hline P12 & 144.29 & 285.9 & 169.88 & 7.47 & 0 \\
\hline P13 & 138 & 416.77 & 291.98 & 9.49 & 0 \\
\hline P14 & 135.17 & 336.34 & 263.55 & 9.61 & 0 \\
\hline P15 & 130.3 & 359.5 & 268.5 & 8.44 & 0 \\
\hline P16 & 122.72 & 361.74 & 153.14 & 6.71 & 0 \\
\hline P17 & 115.41 & 179.24 & 149.15 & 10.5 & 0 \\
\hline P18 & 112.84 & 247.52 & 203.21 & 9.15 & 0.05 \\
\hline P19 & 112.07 & 299.23 & 219.08 & 7.94 & 0 \\
\hline P20 & 111.81 & 343.81 & 194.84 & 10.21 & 0 \\
\hline P21 & 105.14 & 200.69 & 145.01 & 6.16 & 0 \\
\hline P22 & 103.34 & 112.15 & 76.41 & 11.79 & 0 \\
\hline
\end{tabular}

\section{Molecular docking}

For the molecular docking, reported inhibitors of class I sirtuin known as sirtinol and salermide were selected. In this study we have generated four derivatives each of sirtinol and salermide. The structures of ten inhibitors have been shown in Fig. 2. These selected ligands further used for for comparative docking with Sm SirtI and Hs SirtI. The binding affinity of sirtinol and salermide is -10.36 and $-11.25 \mathrm{kcal} / \mathrm{mol}$ with respect to Sm sirtuin1 respectively. For Homo sapiens Sirtuin1 the binding affinity of sirtinol and salermide is -10.77 and $-9.94 \mathrm{kcal} / \mathrm{mol}$ respectively. The sirtinol inhibitor shows slightly higher binding affinity towards Hs Sirt1 with respect to Sm Sirt1. Data of docking results has been summarized in Table 3. 2-D plots of the interaction of ten inhibitors with Human Sirt 1 has been shown in Additional file 1: Figure S3, and the 2-D plots for interaction of ten inhibitors with Schistosoma Sirt1 has been shown in Additional file 1: Figure S4.

\section{ADMET screening}

Druggability and toxicity of ten drug candidates were assessed on the basis of 23 ADMET parameters. The results of ADMET screening have been shown in Additional file 1: Table S1.

\section{Phylogenetic analysis}

The Neighbor-Joining method was used to construct the phylogenetic tree. The minimum branch length lies between S. mansoni and Schistosoma haematobium, Schistosoma japonicum, Clonorchis sinensis $0.00,0.06$ and 0.25 respectively. In Fig. 3 the phylogenetic tree with bootstrap value are shown, that is approximately 100 for similar sequences. The percentage of replicate trees in which the associated taxa clustered together in the bootstrap test $(10,000$ replicates) is shown next to the branches. The p distance model was used to construct the evolutionary distances and branch length represents the number of expected amino acid substitutions per site. On the basis of evolutionary distance of 20 sequences, as mentioned in Table 4, related with sirt1 protein phylogenetic tree was constructed and two major clades were obtained and bootstrap values were also calculated through neighbor-joining method.

\section{Discussion}

Previous studied by Lancelot et al. depicts that transcriptional knockdown of SmSirt1by RNA interference in Schistosoma led to morphological changes in the ovaries characterized by a marked increase in mature oocytes, reiterating the effect of sirtuin inhibitors and suggesting that SmSirt1 may act as principal drug target. The stability of different drug-receptor 
<smiles>CC1CCC2CCCCC2C1CNC1CCCC(NC(C)C(C)C2CCCCC2)C1</smiles>

Salermide<smiles>CC1CCCC([C@@H]2OC3(C(O)C4CCCCC4C3O)[C@H]3[C@H]2C(O)N(C2CCC(Br)CC2)[C@@H]3O)C1</smiles>

sele3<smiles>OC[C@H](N[C@H](O)[C@H]1CCCC[C@@H]1NC[C@@H]1[C@H]2CCCC[C@H]2CC[C@H]1O)C1CCCCC1</smiles>

sirt2<smiles>C[C@H](NC(O)C1CCCCC1NCC1C(O)CCC2CCCCC21)C1CCCCC1</smiles>

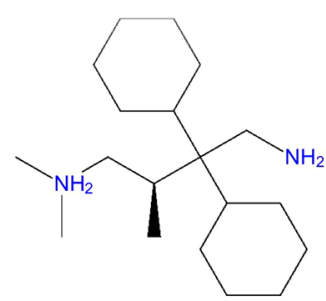

sele1<smiles>NC(O)NNCC1C(O)NC(S)N(C2CCCC(C(F)(F)F)C2)C1O</smiles>

sele4<smiles>OC(O)[C@H](N[C@@H](O)[C@H]1CCCC[C@H]1NC[C@@H]1[C@H]2CCCC[C@H]2CC[C@H]1O)C1CCCCC1</smiles>

sirt3

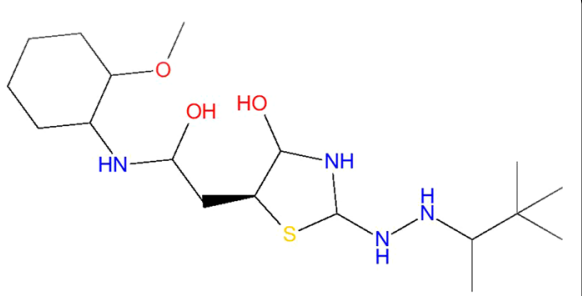

sele2<smiles>OC[C@H](N[C@H](O)[C@H]1CCCC[C@H]1NC[C@@H]1[C@H]2CCCC[C@H]2CC[C@H]1O)C1CCCCC1</smiles>

sirt1

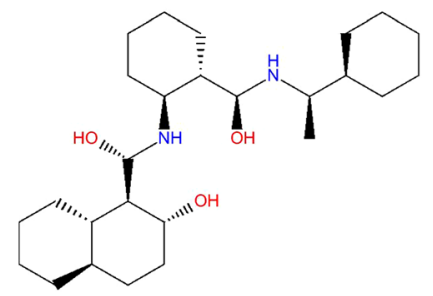

sirt4

Sirtinol

Fig. 2 Selected ligands and their analogs used of molecular docking with hSirt1 and SmSirt1. Sale1-4 represents the analogs of salermide and sirt1-4 represents derivatives of sirtinol

Table 3 Docking results of Human and pathogen Sirtinol1 proteins with 10 drug candidates

\begin{tabular}{|c|c|c|c|c|c|c|}
\hline \multirow[t]{2}{*}{ Ligand } & \multirow{2}{*}{$\begin{array}{l}\text { Binding affinity with } \\
\text { host Sirt1 (kcal/mol) }\end{array}$} & \multirow{2}{*}{$\begin{array}{l}\text { Binding affinity with } \\
\text { pathogen sirt1 (kcal/mol) }\end{array}$} & \multicolumn{2}{|l|}{ Pathogen Sirt1 } & \multicolumn{2}{|l|}{ Host Sirt1 } \\
\hline & & & Ligand efficiency & $\mathrm{IC}(\mathrm{nM})$ & Ligand efficiency & $\mathrm{IC}(\mathrm{nM})$ \\
\hline Sirtinol & -10.77 & -10.36 & -0.35 & 25.57 & -0.36 & 12.78 \\
\hline Sirtinol1 & -9.25 & -11.01 & -0.36 & 8.5 & -0.3 & 165.4 \\
\hline Sirtinol2 & -5.44 & -11 & -0.35 & 8.95 & -0.18 & 0.10333 \\
\hline Sirtinol3 & -5.53 & -9.2 & -0.29 & 180 & -0.17 & 0.08797 \\
\hline Sirtinol4 & -10.51 & -10.33 & -0.33 & 26.95 & -0.34 & 19.9 \\
\hline Salermide & -9.94 & -11.25 & -0.36 & 5.62 & -9.94 & 52.09 \\
\hline Salermide1 & -7.53 & -8.34 & -0.4 & 769.44 & -7.53 & 0.00304 \\
\hline Salermide2 & -8.43 & -5.31 & -0.2 & 127.58 & -8.43 & 666.91 \\
\hline Salermide3 & -8.21 & -6.35 & -0.19 & 22.22 & -8.21 & 960.99 \\
\hline Salermide4 & -8.57 & -9.48 & -0.36 & 112.13 & -8.57 & 519.85 \\
\hline
\end{tabular}




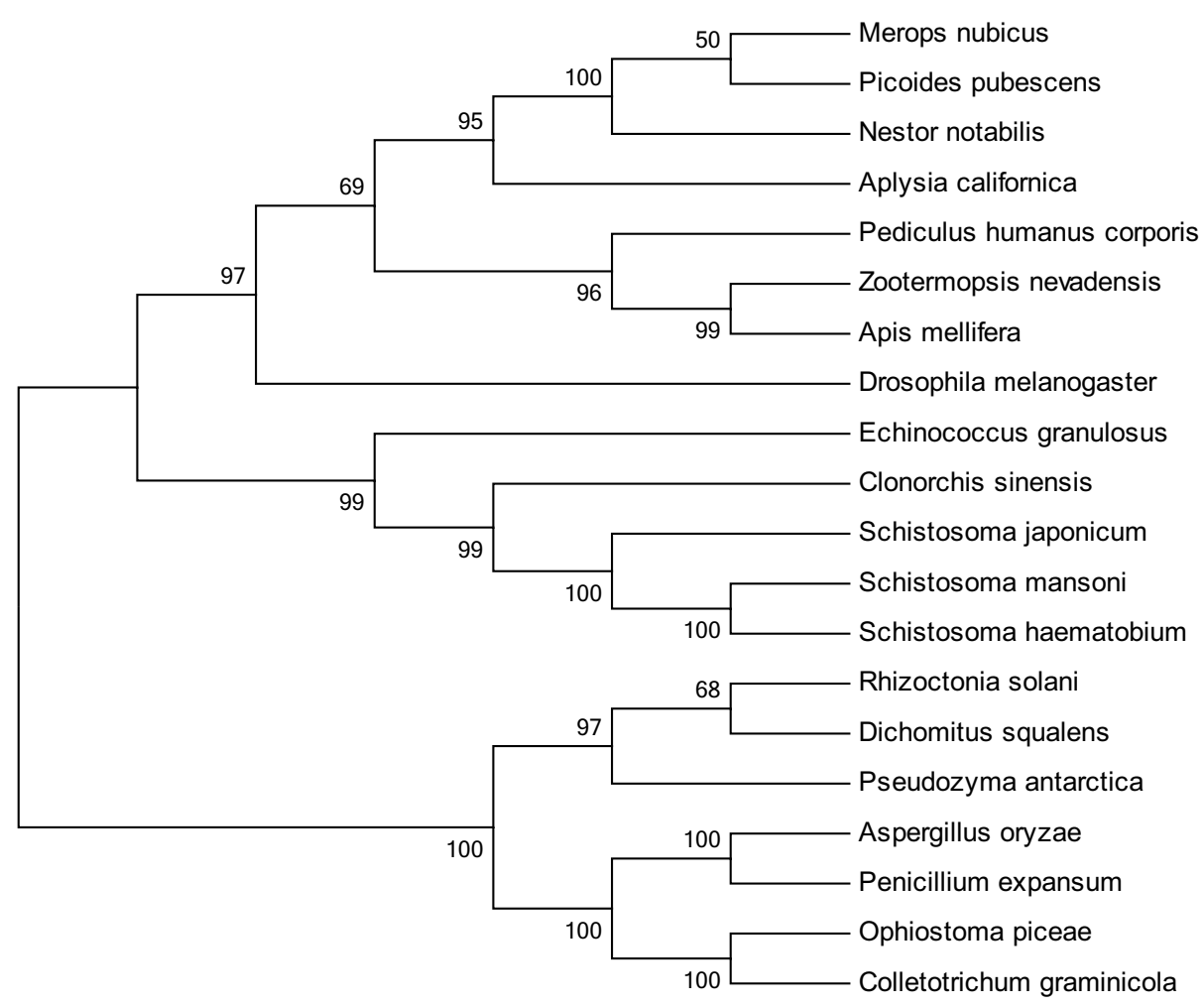

Fig. 3 Phylogenetic analysis of sirtuin1 protein homologues from 20 species was constructed by the neighbor-joining method using the MEGA 5.2 program. Bootstrap values are indicated against each branch. Phylogenetic analysis showed two large clusters of ESX homeobox-1 protein

Table 4 Sirtuin 1 protein of S. mansoni sequence and its different homologues of species with their length and NCBI accession code

\begin{tabular}{llll}
\hline S. No. & Organisms & Acession no. & Length \\
\hline 1 & Schistosoma mansoni & KGB39559.1 & 568 \\
2 & Schistosomajaponicum & CAX8242.1 & 410 \\
3 & Schistosoma haemotobium & KGB39559.1 & 683 \\
4 & Clonorhis sinensis & GAA56043.1 & 600 \\
5 & Echinococcus granulosum & CDS22978.1 & 740 \\
6 & Zootermopsis nevadensis & KDR10345.1 & 882 \\
7 & Pediculas humans coporsis & XP_002432110.1 & 590 \\
8 & Drosophilamelangoaster & AA047879.1 & 483 \\
9 & Rhizoctonia solani & EUC5131.3 & 535 \\
10 & Aspergillus oryzae & XP_001820107.2 & 493 \\
11 & Merops nubicus & KFQ33949.1 & 602 \\
12 & C.higginsianum & EFQ33543.1 & 532 \\
13 & Pencillium expansum & KGO37944.1 & 486 \\
14 & Nestornotabillis & KFQ50963.1 & 602 \\
15 & Ophiostoma piceae & EPE06021.1 & 631 \\
16 & Pseudozyma antarctica & GAK62857.1 & 584 \\
17 & Dichomitus squalens & XP_007362890.1 & 581 \\
18 & Picoides pubescens & KFV72792.1 & 601 \\
19 & Aplysia californica & XP_005095144.1 & 922 \\
20 & Apis mellifera & XP_006569399.1 & 865 \\
\hline
\end{tabular}

complexes on the basis of their docking based binding affinities suggests that salermide and sirtinol both have limited preferential binding affinities for pathogen sirt1 protein in comparison to host homolog. Docking based screening suggests that sirtinol2 and sirtinol3 have considerably higher binding affinity with the parasite receptor (Fig. 4a, b). Sirtinol 1, salermide, salermide1 and salermide4 are having slightly higher binding affinities with pathogen Sirt1 protein.

On the other hand the ADMET screening data suggest that sirtinol1 and salermide due to having AMES toxicity may not be considered as drug candidate. Salermide1 is a predicted carcinogen in the AMES tat and salermide4 is a P-glycoprotein inhibitor. Sirtinol3 has been predicted as a drug candidate which has neither blood-brain barrier crossing nor human intestine absorption capability. So, this may have prospects of being used as injection drugs that are directly mixed in blood. Further sirtinol2 is also a promising drug candidate for treatment of Schistosmiasis. Though sirtinol2 is not having blood brain carrier permeability but it is not a major issue as the location of the pathogen in host is intestine. 
a

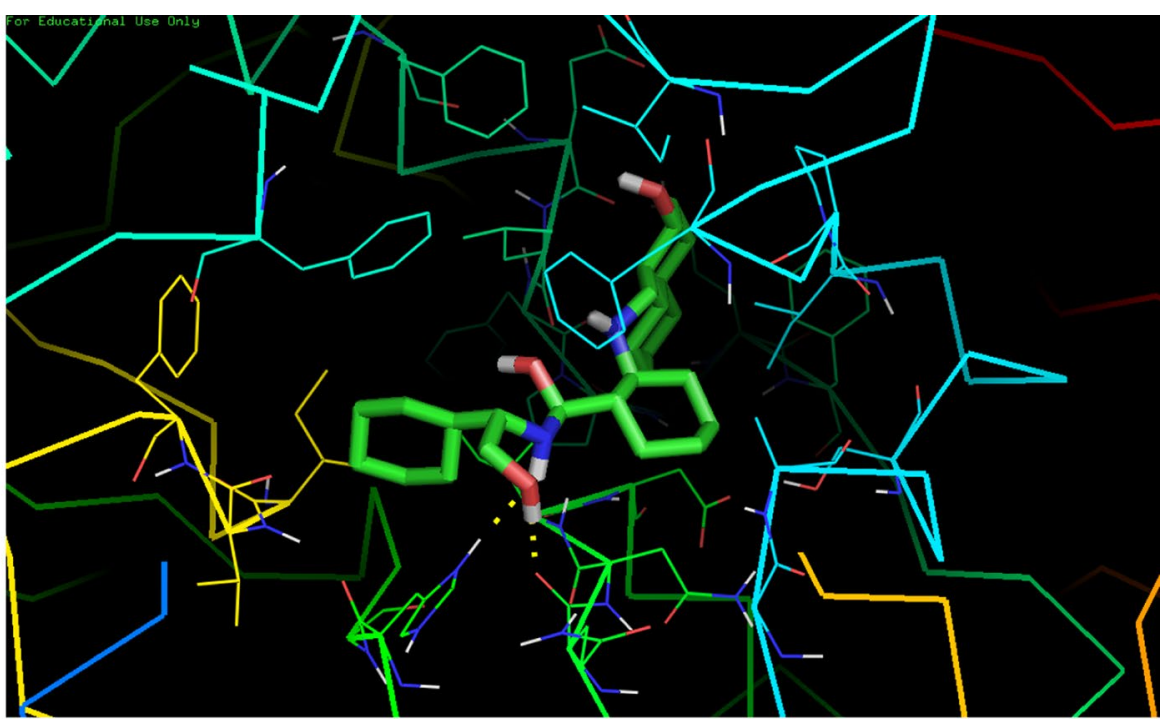

b

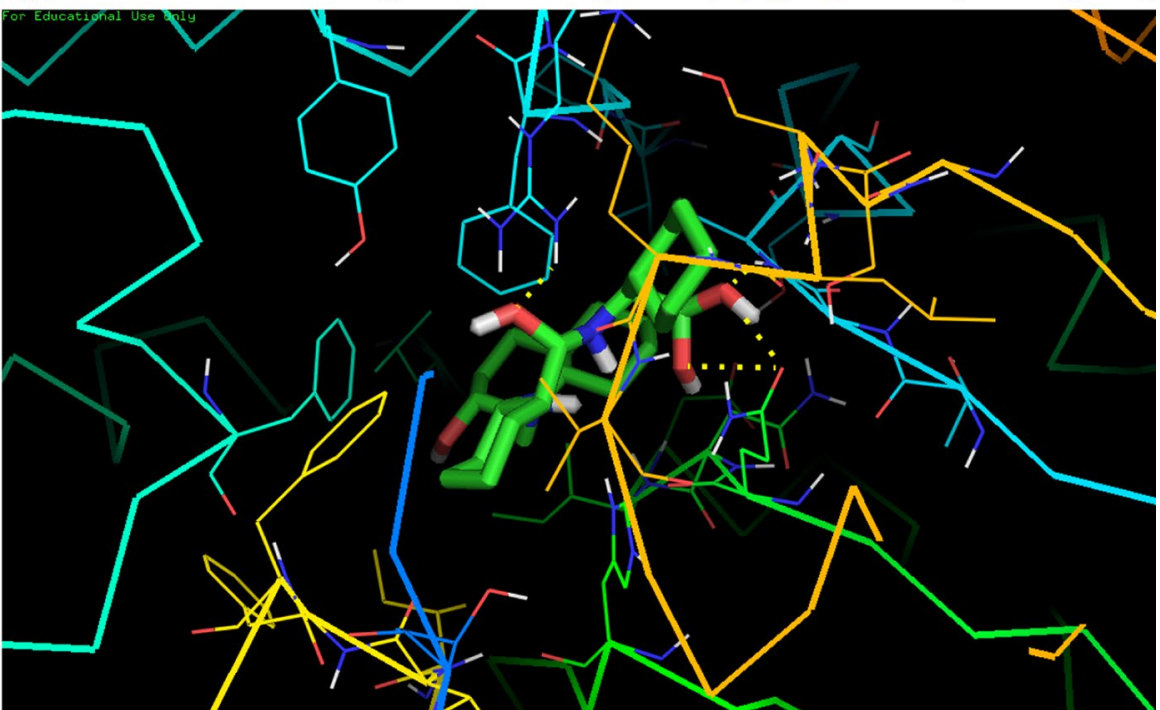

Fig. 4 a Interaction between sirt2 inhibitor and pathogen sirtuin1 protein. $\mathbf{b}$ Interaction between sirt3 inhibitor and pathogen sirtuin1 protein

\section{Conclusions}

Aim of this study was to design and screen new drugs for treatment of Schistosomiasis which is a disease prevalent in tropical regions of the world. We have screened two drug candidates named as sirtinol 2 and sirtinol3, both are derived for the structures of sirtinol, a reported inhibitor of Schistosoma mansoni. There is need to search the druggability of the screened drug candidates which may prove to be promising in the way of providing low cost and safe drugs to the needy population in the society. This study will trigger the drug development and prospective clinical trial process for schistosomiasis may prove to be a milestone to new drug discovery.

\section{Additional file}

Additional file 1: Figure S1. Ramachandran plot of modeled Sirt1. Figure S2. Z-score plot shows reliability of modeled structure. The black dot represents Z-score of modeled Sirtuin1 protein of S. mansoni. Figure S3. 2D plots of all the inhibitors human Sirt1. Here 1 represents salermide, 2 with sirtinol, 3, 4, 5 and 6 with sale1, sale2, sale3, sale4 and sale5 and 7, 8, 9 and 10 with sirt1, sirt2, sirt 3 and sirt4. Figure S4. 2D plots of all the inhibitors with Schistosoma Sirt1. Here 1 represents salermide, 2 with sirtinol, 3 , 4, 5 and 6 with sale1, sale2, sale3, sale 4 and sale 5 and $7,8,9$ and 10 with sirt1, sirt2, sirt3 and sirt4. Table S1. ADMET screening results.

\section{Authors' contributions}

RS, BSY, SS and AM performed the experiments and manuscript writing. PNP helped in data analysis and manuscript writing. AM conceived the project. All authors read and approved the final manuscript. 


\section{Author details}

${ }^{1}$ Institute of Interdisciplinary Studies, Center of Bioinformatics, Nehru Science Center, University of Allahabad, Allahabad 211002 , India. ${ }^{2}$ Department of Biotechnology, Motilal Nehru National Institute of Technology Allahabad, Allahabad 211004, India. ${ }^{3}$ Department of Mathematics, University of Allahabad, Allahabad 211002, India.

\section{Acknowledgements}

BSY is thankful to DST INSPIRE for PhD fellowship. AM is thankful to MNNIT Allahabad for the computational facility.

\section{Competing interests}

The authors declare that they have no competing interests.

Received: 26 November 2015 Accepted: 17 February 2016 Published online: 07 March 2016

\section{References}

Altschul SF, Gish W, Miller W, Myers EW, Lipman DJ (1990) Basic local alignment search tool. J Mol Biol 215(3):403-410

Altschul SF, Madden TL, Schäffer AA, Zhang J, Zhang Z, Miller W, Lipman DJ (1997) Gapped BLAST and PSI-BLAST: a new generation of protein database search programs. Nucleic Acids Res 25(17):3389-3402

Cheng F, Li W, Zhou Y, Shen J, Wu Z, Liu G, Lee PW, Tang Y (2012) admetSAR: a comprehensive source and free tool for assessment of chemical ADMET properties. J Chem Inf Model 52(11):3099-3105

Cioli D, Pica-Mattoccia L, Archer S (1993) Drug resistance in schistosomes. Parasitol Today 9(5):162-166

Doenhoff MJ, Cioli D, Utzinger J (2008) Praziquantel: mechanisms of action, resistance and new derivatives for schistosomiasis. Curr Opin Infect Dis 21(6):659-667

Dömling A, Khoury K (2010) Praziquantel and schistosomiasis. ChemMedChem 5(9):1420-1434

Fallon PG, Doenhoff MJ (1994) Drug-resistant schistosomiasis: resistance to praziquantel and oxamniquine induced in Schistosoma mansoni in mice is drug specific. Am J Trop Med Hyg 51(1):83-88

Felsenstein J (1985) Confidence limits on phylogenies: an approach using the bootstrap. Evolution 39(4):783-791

Fenwick A, Webster JP, Bosque-Oliva E, Blair L, Fleming F, Zhang Y, Garba A, Stothard J, Gabrielli AF, Clements A (2009) The Schistosomiasis control initiative (SCI): rationale, development and implementation from 2002-2008. Parasitology 136(13):1719-1730

Frye RA (2000) Phylogenetic classification of prokaryotic and eukaryotic Sir2like proteins. Biochem Biophys Res Commun 273(2):793-798

Hall TA (1999) BioEdit: a user-friendly biological sequence alignment editor and analysis program for Windows 95/98/NT. In: Nucleic acids symposium series. 95-98
Lancelot J, Caby S, Dubois-Abdesselem F, Vanderstraete M, Trolet J, Oliveira G, Bracher F, Jung M, Pierce RJ (2013) Schistosoma mansoni sirtuins: characterization and potential as chemotherapeutic targets. PLoS Negl Trop Dis 7:e2428

Lara E, Mai A, Calvanese V, Altucci L, Lopez-Nieva P, Martinez-Chantar M, Varela-Rey M, Rotili D, Nebbioso A, Ropero S (2009) Salermide, a sirtuin inhibitor with a strong cancer-specific proapoptotic effect. Oncogene 28(6):781-791

Larkin MA, Blackshields G, Brown N, Chenna R, McGettigan PA, McWilliam H, Valentin F, Wallace IM, Wilm A, Lopez R (2007) Clustal W and Clustal X version 2.0. Bioinformatics 23(21):2947-2948

Martí-Renom MA, Stuart AC, Fiser A, Sánchez R, Melo F, Šali A (2000) Comparative protein structure modeling of genes and genomes. Annu Rev Biophys Biomol Struct 29(1):291-325

Melman SD, Steinauer ML, Cunningham C, Kubatko LS, Mwangi IN, Wynn NB, Mutuku MW, Karanja D, Colley DG, Black CL (2009) Reduced susceptibility to praziquantel among naturally occurring Kenyan isolates of Schistosoma mansoni. PLoS Negl Trop Dis 3(8):e504

Morris GM, Huey R, Lindstrom W, Sanner MF, Belew RK, Goodsell DS, Olson AJ (2009) AutoDock4 and AutoDockTools4: automated docking with selective receptor flexibility. J Comput Chem 30(16):2785-2791

Pierce JR, Dubois-Abdesselem F, Lancelot J, Andrade L, Oliveira G (2012) Targeting schistosome histone modifying enzymes for drug development. Curr Pharm Des 18(24):3567-3578

Religa AA, Waters AP (2012) Sirtuins of parasitic protozoa: in search of function (s). Mol Biochem Parasitol 185(2):71-88

Saitou N, Nei M (1987) The neighbor-joining method: a new method for reconstructing phylogenetic trees. Mol Biol Evol 4(4):406-425

Šali A, Blundell TL (1993) Comparative protein modelling by satisfaction of spatial restraints. J Mol Biol 234(3):779-815

Singh R, Pandey PN (2015) Molecular docking and molecular dynamics study on SmHDAC1 to identify potential lead compounds against Schistosomiasis. Mol Biol Rep 42(3):689-698

Singh R, Singh S, Pandey PN (2015) In-silico analysis of sirt2 from Schistosoma mansoni: structures, conformations, and interactions with inhibitors. J Biomol Struct Dyn. doi:10.1080/07391102.2015.1065205

Tamura K, Peterson D, Peterson N, Stecher G, Nei M, Kumar S (2011) MEGA5: molecular evolutionary genetics analysis using maximum likelihood, evolutionary distance, and maximum parsimony methods. Mol Biol Evol 28(10):2731-2739

Vergnes B, Sereno D, Madjidian-Sereno N, Lemesre J-L, Ouaissi A (2002) Cytoplasmic SIR2 homologue overexpression promotes survival of Leishmania parasites by preventing programmed cell death. Gene 296(1):139-150

Volkamer A, Kuhn D, Rippmann F, Rarey M (2012) DoGSiteScorer: a web server for automatic binding site prediction, analysis and druggability assessment. Bioinformatics 28(15):2074-2075

Wiederstein M, Sippl MJ (2007) ProSA-web: interactive web service for the recognition of errors in three-dimensional structures of proteins. Nucleic Acids Res 35(suppl 2):W407-W410

\section{Submit your manuscript to a SpringerOpen ${ }^{\circ}$ journal and benefit from:}

- Convenient online submission

- Rigorous peer review

- Immediate publication on acceptance

- Open access: articles freely available online

- High visibility within the field

- Retaining the copyright to your article

Submit your next manuscript at $>$ springeropen.com 